\title{
A GENERAL EXTRAPOLATION THEOREM FOR ABSOLUTELY SUMMING OPERATORS
}

\author{
D. PELlEGRINO, J. SANTOS, AND J. B. SEOANE-SEPÚLVEDA
}

\begin{abstract}
In this note we prove a general version of the Extrapolation Theorem, extending the classical linear extrapolation theorem due to B. Maurey. Our result shows, in particular, that the operators involved do not need to be linear.
\end{abstract}

\section{INTRODUCTION AND BACKGROUND}

The notion of absolutely $(p ; q)$-summing linear operators is due to A. Pietsch 18 and B. Mitiagin and A. Pełczyński [14, inspired by previous works of A. Grothendieck. The nonlinear theory of absolutely summing operators was initiated by A. Pietsch and a complete nonlinear approach was introduced by M.C. Matos [12.

Let $X, Y$ be Banach spaces over a fixed scalar field $\mathbb{K}=\mathbb{R}$ or $\mathbb{C}$; for $1 \leq p<\infty$, denote by $\ell_{p, w}(X)$ the space of all sequences $\left(x_{j}\right)_{j=1}^{\infty}$ in $X$ such that $\left(\varphi\left(x_{j}\right)\right)_{j=1}^{\infty} \in \ell_{p}$ for every $\varphi$ in the topological dual of $X$ (represented by $X^{*}$ ). Let $\mathcal{L}$ be the class of all continuous linear operators between Banach spaces over $\mathbb{K}$, i.e., $\mathcal{L}=\bigcup_{X, Y} \mathcal{L}(X ; Y)$, where $X, Y$ run over all Banach spaces over $\mathbb{K}$.

A continuous linear operator $u: X \rightarrow Y$ is absolutely $p$-summing (we write $u \in \Pi_{p}(X ; Y)$ ) if $\left(u\left(x_{j}\right)\right)_{j=1}^{\infty} \in$ $\ell_{p}(Y)$ whenever $\left(x_{j}\right)_{j=1}^{\infty} \in \ell_{p, w}(X)$. For details we refer to the classical monograph [8] and to [4, 9] for more recent results.

An important result in the theory of absolutely summing linear operators is the Extrapolation Theorem due to B. Maurey [13]:

Theorem 1.1 (Extrapolation Theorem). Let $1<r<p<\infty$ and let $X$ be a Banach space. If

$$
\Pi_{p}\left(X ; \ell_{p}\right)=\Pi_{r}\left(X ; \ell_{p}\right)
$$

then, for any Banach space $Y$,

$$
\Pi_{p}(X ; Y)=\Pi_{1}(X ; Y) .
$$

In the present paper we show that this Extrapolation Theorem holds in a much more general form, where the linearity of the operators is not needed. For example, as a very particular case of our main result we generalize the Extrapolation Theorem in the following way:

Let $\mathbb{K}$ be a fixed scalar field $\mathbb{R}$ or $\mathbb{C}, 1 \leq p<\infty$ and $\mathcal{F}$ a non-void family of maps, $\mathcal{F} \subset \bigcup_{X, Y} X^{Y}$, where $X$ and $Y$ run over all Banach spaces over $\mathbb{K}$. We say that $f \in \mathcal{F}$ is absolutely $p$-summing if there is a $C \geq 0$ so that

$$
\left(\sum_{j=1}^{m}\left\|f\left(x_{j}\right)\right\|^{p}\right)^{1 / p} \leq C\left(\sup _{\varphi \in B_{E^{*}}} \sum_{j=1}^{m}\left|\varphi\left(x_{j}\right)\right|^{p}\right)^{1 / p}
$$

for every positive integer $m$ and every $x_{1}, \ldots, x_{m} \in X$. Let

$$
\Pi_{\mathcal{F}, p}(X ; Y)=\{f \in \mathcal{F}: f: X \rightarrow Y \text { is absolutely } p \text {-summing }\} .
$$

D. Pellegrino was supported by CNPq Grant 301237/2009-3. J. B. Seoane-Sepúlveda was supported by the Spanish Ministry of Science and Innovation, grant MTM2009-07848. 
Note that $\Pi_{\mathcal{F}, p}(X ; Y)$ is a vector space and the infimum of the $C$ 's satisfying (1.1) is a norm for $\Pi_{\mathcal{F}, p}(X ; Y)$ denoted by $\pi_{\mathcal{F}, p}$. If $\left(\Pi_{\mathcal{F}, p}(X ; Y), \pi_{\mathcal{F}, p}\right)$ is complete and $\mathcal{F}$ is so that $T \circ f \in \mathcal{F}$ whenever $T \in \mathcal{L}(Y ; Z)$ and $f \in \Pi_{\mathcal{F}, p}(X ; Y)$, then, as a consequence of our main result, we have the following theorem:

Theorem 1.2 (Nonlinear Extrapolation Theorem). Let $1<r<p<\infty$ and let $X$ be a Banach space. If

$$
\Pi_{\mathcal{F}, p}\left(X ; \ell_{p}\right)=\Pi_{\mathcal{F}, r}\left(X ; \ell_{p}\right),
$$

then, for any Banach space $Y$,

$$
\Pi_{\mathcal{F}, p}(X ; Y)=\Pi_{\mathcal{F}, 1}(X ; Y) .
$$

In particular, when $\mathcal{F}=\mathcal{L}$ we recover Maurey's Extrapolation Theorem.

Our general result (Theorem 3.1) also furnishes, as a simple particular case, a recent Extrapolation Theorem for Lipschitz summing mappings, due to D. Chen and B. Zheng [7.

One of the pillars of the theory of absolutely summing operators is the famous Pietsch Domination Theorem which asserts that a continuous linear operator $u: X \rightarrow Y$ is absolutely $p$-summing if, and only if, there is a regular probability measure $\mu$ in the Borel sets of $B_{X^{*}}$ (with the weak-star topology) and a constant $C \geq 0$ such that

for all $x \in X$.

$$
\|u(x)\| \leq C\left(\int_{B_{X^{*}}}|\varphi(x)|^{p} d \mu(\varphi)\right)^{1 / p}
$$

Very recently, a series of works ([2, 3, 15, 16, 17]) on Pietsch Domination-Factorization Theorem have shown that Pietsch Domination Theorem in fact needs almost no linear structure and a quite general version is valid (see Theorem 1.3 below) which has shown to be very useful in different contexts (1, 6]).

Let $X, Y$ and $E$ be (arbitrary) non-void sets, $\mathcal{H}(X ; Y)$ be a non-void family of mappings from $X$ to $Y$, $G$ be a Banach space and $K$ be a compact Hausdorff topological space. Let

$$
R: K \times E \times G \longrightarrow[0, \infty) \text { and } S: \mathcal{H}(X ; Y) \times E \times G \longrightarrow[0, \infty)
$$

be arbitrary mappings and $1 \leq t<\infty$. According to [3, 16] a mapping $f \in \mathcal{H}(X ; Y)$ is $R S$-abstract $t$-summing if there is a constant $C \geq 0$ so that

$$
\left(\sum_{j=1}^{m} S\left(f, x_{j}, b_{j}\right)^{t}\right)^{\frac{1}{t}} \leq C \sup _{\varphi \in K}\left(\sum_{j=1}^{m} R\left(\varphi, x_{j}, b_{j}\right)^{t}\right)^{\frac{1}{t}},
$$

for all $x_{1}, \ldots, x_{m} \in E, b_{1}, \ldots, b_{m} \in G$ and $m \in \mathbb{N}$. We define

$$
\mathcal{H}_{R S, t}(X ; Y)=\{f \in \mathcal{H}(X ; Y): f \text { is } R S \text {-abstract } t \text {-summing }\} .
$$

Suppose that $R$ is so that the mapping

$$
R_{x, b}: K \longrightarrow[0, \infty) \text { defined by } R_{x, b}(\varphi)=R(\varphi, x, b)
$$

is continuous for every $x \in E$ and $b \in G$. The Pietsch Domination Theorem from [16] reads as follows:

Theorem 1.3. Suppose that $S$ is arbitrary, $R$ satisfies (1.3) and let $1 \leq p<\infty$. A map $h \in \mathcal{H}(X ; Y)$ is $R S$-abstract p-summing if and only if there is a constant $C \geq 0$ and a Borel probability measure $\mu$ on $K$ such that

$$
S(h, e, b) \leq C\|R(\cdot, e, b)\|_{L_{p}(K, \mu)}
$$

for all $e \in E$ and $b \in G$.

This general approach recovers several Pietsch Domination type theorems (see [3]) and also rapidly found applications in different contexts (see [1, 6]). It is worth mentioning that the recent interesting version of the Pietsch Domination Theorem for Lipschitz $(p ; q ; r)$-summing operators proved in [5, Theorem 5.4 (a) $\Rightarrow(\mathrm{b})$ ] can also be obtained as a simple application of the general result from 17 . 
In the next section we present the main result of this note, the general Extrapolation Theorem (in the lines of the abstract setting of Theorem 1.3) which, as the general Pietsch Domination Theorem, does not need any linear setting. In the final section we show how the general Extrapolation Theorem can be applied.

\section{A NEW ABSTRACT SETTING}

In this section we build the environment needed for the proof of the general Extrapolation Theorem. We keep the notation from the previous section. Also, let $p, r$ be such that $1<r<p<\infty$. Suppose that $X$ is a topological space, $E=X \times X$ and $K$ is a compact Hausdorff space such that $X$ is continuously embedded in $C(K)$. We denote by $\mathcal{P}(K)$ the collection of all regular Borel probability measures on $K$. For any $\mu \in \mathcal{P}(K)$, let $j_{\mu}: X \rightarrow L_{p}(\mu):=L_{p}(K, \mu)$ denote the composition of the inclusion $X \rightarrow C(K)$ with the canonical map $C(K) \rightarrow L_{p}(\mu)$. We will keep the notation, terminology and assumptions from above. Also, suppose that

(1) For all $t \in\{1, r, p\}$ and all Banach spaces $Y, \mathcal{H}_{R S, t}(X ; Y)$ is a vector space and the infimum of the $C^{\prime}$ 's satisfying (1.2) is a (complete) norm for $\mathcal{H}_{R S, t}(X ; Y)$, denoted by $\pi_{R S, t}(\cdot)$.

(2) For all Banach spaces $Y, Z$, if $h \in \mathcal{H}(X ; Y)$ and $T: Y \rightarrow Z$ is a bounded linear operator, then

$$
S(T \circ h,(x, q), b) \leq\|T\| S(h,(x, q), b) .
$$

(3) $j_{\mu} \in \mathcal{H}\left(X ; L_{p}(\mu)\right)$ and

$$
S\left(j_{\mu},(x, q), b\right)=\|R(\cdot,(x, q), b)\|_{L_{p}(K, \mu)}
$$

for all $((x, q), b) \in E \times G$.

(4) If $\left\{j_{\mu}\left(x_{1}\right), \ldots, j_{\mu}\left(x_{m}\right), j_{\mu}\left(q_{1}\right), \ldots j_{\mu}\left(q_{m}\right)\right\}$ is contained in a finite-dimensional subspace $F$ of $L_{p}(\mu)$ and $p_{F}: L_{p}(\mu) \rightarrow F$ denotes the canonical projection, then

$$
\sum_{j=1}^{m} S\left(j_{\mu},\left(x_{j}, q_{j}\right), b_{j}\right)^{r}=\sum_{j=1}^{m} S\left(p_{F} \circ j_{\mu},\left(x_{j}, q_{j}\right), b_{j}\right)^{r} .
$$

Also $p_{F} \circ j_{\mu} \in \mathcal{H}_{R S, p}(X ; F)$ and there is a $C_{1}>0$ (not depending on $F$ ) so that

$$
\pi_{R S, p}\left(p_{F} \circ j_{\mu}\right) \leq C_{1} \pi_{R S, p}\left(j_{\mu}\right) .
$$

(5) If $\mathcal{H}_{R S, p}\left(X ; \ell_{p}\right)=\mathcal{H}_{R S, r}\left(X ; \ell_{p}\right)$ and $\pi_{\mathcal{H}_{R S, r}}(v) \leq c \cdot \pi_{\mathcal{H}_{R S, p}}(v)$ for all $v \in \mathcal{H}_{R S, p}\left(X ; \ell_{p}\right)$, then

$$
\mathcal{H}_{R S, p}\left(X ; \ell_{p}^{m}\right)=\mathcal{H}_{R S, r}\left(X ; \ell_{p}^{m}\right)
$$

and

$$
\pi_{\mathcal{H}_{R S, r}}(v) \leq c \cdot \pi_{\mathcal{H}_{R S, p}}(v)
$$

for all $v \in \mathcal{H}_{R S, p}\left(X ; \ell_{p}^{m}\right)$ and all positive integers $m$.

It may seem that we have too many hypotheses but we recall that we are working in a very abstract setting and for this reason it is natural to need to introduce some hypotheses. Moreover, a careful examination shows that the above hypotheses are quite natural.

\section{The general Extrapolation Theorem}

Following the assumptions and terminology of the previous sections we can state and prove our main result:

Theorem 3.1. Let $1<r<p<\infty$. If

$$
\mathcal{H}_{R S, p}\left(X ; \ell_{p}\right)=\mathcal{H}_{R S, r}\left(X ; \ell_{p}\right)
$$

then, for any Banach space $Y$,

$$
\mathcal{H}_{R S, p}(X ; Y)=\mathcal{H}_{R S, 1}(X ; Y)
$$


Proof. From Theorem 1.3, using the monotonicity of the $L_{p}$ norms we have

$$
\mathcal{H}_{R S, 1}(X ; Y) \subset \mathcal{H}_{R S, p}(X ; Y) \text {. }
$$

For the converse inclusion, it suffices to prove that, regardless of the Banach space $Y$, there is $C>0$ such that for each $h \in \mathcal{H}_{R S, p}(X ; Y)$, we have

$$
\pi_{R S, 1}(h) \leq C \cdot \pi_{R S, p}(h) .
$$

Since $\mathcal{H}_{R S, p}\left(X ; \ell_{p}\right)=\mathcal{H}_{R S, r}\left(X ; \ell_{p}\right)$, it follows from the Open Mapping Theorem and (1) that there is $c>0$ such that

$$
\pi_{\mathcal{H}_{R S, r}}(v) \leq c \cdot \pi_{\mathcal{H}_{R S, p}}(v)
$$

for all $v \in \mathcal{H}_{R S, p}\left(X ; \ell_{p}\right)$. Let $\mu \in \mathcal{P}(K)$; since $L_{p}(K, \mu)$ is an $\mathcal{L}_{p, \lambda}$ space for all $\lambda>1$ (see [8, Theorem 3.2]), we can assert that for each $\left(x_{i}\right)_{1 \leq i \leq m},\left(q_{i}\right)_{1 \leq i \leq m}$ in $X$, the subspace of $L_{p}(K, \mu)$ generated by $\left\{j_{\mu}\left(x_{1}\right), \ldots, j_{\mu}\left(x_{m}\right), j_{\mu}\left(q_{1}\right), \ldots, j_{\mu}\left(q_{m}\right)\right\}$ embeds $\lambda$-isomorphically into $\ell_{p}$. More precisely,

$$
\operatorname{span}\left\{j_{\mu}\left(x_{1}\right), \ldots, j_{\mu}\left(x_{m}\right), j_{\mu}\left(q_{1}\right), \ldots, j_{\mu}\left(q_{m}\right)\right\}
$$

is contained in a subspace $F$ of $L_{p}(K, \mu)$ for which there is an isomorphism $T: F \rightarrow \ell_{p}^{\operatorname{dim} F}$ with

$$
\|T\|\left\|T^{-1}\right\|<\lambda
$$

From (3) $j_{\mu}$ is $R S$-abstract $p$-summing and

$$
\pi_{R S, p}\left(j_{\mu}\right) \leq 1
$$

Hence, from (3.3) and (2.2) we have

$$
\pi_{R S, p}\left(p_{F} \circ j_{\mu}\right) \leq C_{1}
$$

and, from (2), it follows that $T \circ p_{F} \circ j_{\mu} \in \mathcal{H}_{R S, p}\left(X ; \ell_{p}^{\operatorname{dim} F}\right)$. Finally, from (5) and (3.1) we can assert that

$$
T \circ p_{F} \circ j_{\mu} \in \mathcal{H}_{R S, r}\left(X ; \ell_{p}^{\operatorname{dim} F}\right) .
$$

and

$$
\pi_{\mathcal{H}_{R S, r}}\left(T \circ p_{F} \circ j_{\mu}\right) \leq c \cdot \pi_{\mathcal{H}_{R S, p}}\left(T \circ p_{F} \circ j_{\mu}\right) .
$$


We thus have

$$
\begin{aligned}
& \left(\sum_{j=1}^{m} S\left(j_{\mu},\left(x_{j}, q_{j}\right), b_{j}\right)^{r}\right)^{1 / r} \stackrel{2.1}{=}\left(\sum_{j=1}^{m} S\left(T^{-1} \circ T \circ p_{F} \circ j_{\mu},\left(x_{j}, q_{j}\right), b_{j}\right)^{r}\right)^{1 / r} \\
& \stackrel{(2)}{\leq}\left\|T^{-1}\right\|\left(\sum_{j=1}^{m} S\left(T \circ p_{F} \circ j_{\mu},\left(x_{j}, q_{j}\right), b_{j}\right)^{r}\right)^{1 / r} \\
& \stackrel{3.5}{\leq}\left\|T^{-1}\right\| \pi_{\mathcal{H}_{R S, r}}\left(T \circ p_{F} \circ j_{\mu}\right) \sup _{\varphi \in K}\left(\sum_{j=1}^{m} R\left(\varphi,\left(x_{j}, q_{j}\right), b_{j}\right)^{r}\right)^{1 / r} \\
& \stackrel{\text { 3.6) }}{\leq} c \cdot\left\|T^{-1}\right\| \pi_{\mathcal{H}}\left(T \circ p_{F} \circ j_{\mu}\right) \sup _{\varphi \in K}\left(\sum_{j=1}^{m} R\left(\varphi,\left(x_{j}, q_{j}\right), b_{j}\right)^{r}\right)^{1 / r} \\
& \stackrel{(2)}{\leq} c \cdot\left\|T^{-1}\right\|\|T\| \pi_{\mathcal{H}_{R S, p}}\left(p_{F} \circ j_{\mu}\right) \sup _{\varphi \in K}\left(\sum_{j=1}^{m} R\left(\varphi,\left(x_{j}, q_{j}\right), b_{j}\right)^{r}\right)^{1 / r} \\
& \stackrel{3.21}{\leq} c \cdot \lambda \cdot C_{1} \sup _{\varphi \in K}\left(\sum_{j=1}^{m} R\left(\varphi,\left(x_{j}, q_{j}\right), b_{j}\right)^{r}\right)^{1 / r}
\end{aligned}
$$

So

$$
\pi_{\mathcal{H}_{R S, r}}\left(j_{\mu}\right) \leq c \cdot C_{1} \cdot \lambda
$$

and, from Theorem 1.3 , there is $\hat{\mu} \in \mathcal{P}(K)$

$$
S\left(j_{\mu},(x, q), b\right) \leq c \cdot C_{1} \cdot \lambda\left(\int_{K} R(\varphi,(x, q), b)^{r} d \hat{\mu}(\varphi)\right)^{1 / r},
$$

for all $((x, q), b) \in E \times G$. Thus,

$$
\|R(\cdot,(x, q), b)\|_{L_{p}(K, \mu)} \stackrel{(3)}{=} S\left(j_{\mu},(x, q), b\right) \stackrel{3.7}{\leq} c \cdot C_{1} \cdot \lambda\|R(\cdot,(x, q), b)\|_{L_{r}(K, \hat{\mu})},
$$

for all $((x, q), b) \in E \times G$.

Now let $h \in \mathcal{H}_{R S, p}(X ; Y)$. From Theorem 1.3 there is a $\mu_{0} \in \mathcal{P}(K)$ such that

$$
S(h,(x, q), b) \leq \pi_{R S, p}(h)\left(\int_{K} R(\varphi,(x, q), b)^{p} d \mu_{0}(\varphi)\right)^{1 / p}=\pi_{R S, p}(h)\|R(\cdot,(x, q), b)\|_{L_{p}\left(K, \mu_{0}\right)}
$$

for all $((x, q), b) \in E \times G$. Now it is enough to show that

$$
\|R(\cdot,(x, q), b)\|_{L_{p}\left(K, \mu_{0}\right)} \leq C\|R(\cdot,(x, q), b)\|_{L_{1}(K, \bar{\mu})}
$$

for some $\bar{\mu} \in \mathcal{P}(K)$ and some constant $C$ depending only on $X$; the rest of proof follows the lines of Maurey's original argument. Starting with $\mu_{0}$, define $\left(\mu_{n}\right)_{n=0}^{\infty}$ in $\mathcal{P}(K)$ by setting $\mu_{n+1}=\hat{\mu}_{n}, n=0,1, \ldots$. and define

$$
\bar{\mu}=\sum_{n=0}^{\infty} 2^{-n-1} \mu_{n}
$$

Then $\bar{\mu} \in \mathcal{P}(K)$ and, since $1<r<p$, there exists a $\theta \in(0,1)$ so that

$$
\frac{1}{r}=\theta+\frac{1-\theta}{p}
$$


Using Littlewood's Inequality (see [11, p. 55]), we get

$$
\begin{aligned}
\|R(\cdot,(x, q), b)\|_{L_{r}\left(K, \mu_{n}\right)} & =\left(\int_{K} R(\varphi,(x, q), b)^{r} d \mu_{n}(\varphi)\right)^{1 / r} \\
& \leq\left(\int_{K} R(\varphi,(x, q), b) d \mu_{n}(\varphi)\right)^{\theta}\left(\int_{K} R(\varphi,(x, q), b)^{p} d \mu_{n}(\varphi)\right)^{\frac{1-\theta}{p}} \\
& =\|R(\cdot,(x, q), b)\|_{L_{1}\left(K, \mu_{n}\right)}^{\theta}\|R(\cdot,(x, q), b)\|_{L_{p}\left(K, \mu_{n}\right)}^{1-\theta} .
\end{aligned}
$$

Then,

$$
\begin{aligned}
& \sum_{n=0}^{\infty} 2^{-n-1}\|R(\cdot,(x, q), b)\|_{L_{p}\left(K, \mu_{n}\right)} \stackrel{3.8}{\leq} c \cdot C_{1} \cdot \lambda \sum_{n=0}^{\infty} 2^{-n-1}\|R(\cdot,(x, q), b)\|_{L_{r}\left(K, \hat{\mu}_{n}\right)} \\
& =c \cdot C_{1} \cdot \lambda \sum_{n=0}^{\infty} 2^{-n-1}\|R(\cdot,(x, q), b)\|_{L_{r}\left(K, \mu_{n+1}\right)} \\
& \stackrel{3.9}{\leq} c \cdot C_{1} \cdot \lambda \sum_{n=0}^{\infty} 2^{-n-1}\|R(\cdot,(x, q), b)\|_{L_{1}\left(K, \mu_{n+1}\right)}^{\theta}\|R(\cdot,(x, q), b)\|_{L_{p}\left(K, \mu_{n+1}\right)}^{1-\theta} \\
& =c \cdot C_{1} \cdot \lambda \sum_{n=0}^{\infty}\left(2^{-n-1}\right)^{\theta}\|R(\cdot,(x, q), b)\|_{L_{1}\left(K, \mu_{n+1}\right)}^{\theta}\left(2^{-n-1}\right)^{1-\theta}\|R(\cdot,(x, q), b)\|_{L_{p}\left(K, \mu_{n+1}\right)}^{1-\theta} \\
& =c \cdot C_{1} \cdot \lambda \sum_{n=0}^{\infty}\left(2^{-n-1}\|R(\cdot,(x, q), b)\|_{L_{1}\left(K, \mu_{n+1}\right)}\right)^{\theta}\left(2^{-n-1}\|R(\cdot,(x, q), b)\|_{L_{p}\left(K, \mu_{n+1}\right)}\right)^{1-\theta} \\
& \stackrel{(*)}{\leq} c \cdot C_{1} \cdot \lambda\left(\sum_{n=0}^{\infty} 2^{-n-1}\|R(\cdot,(x, q), b)\|_{L_{1}\left(K, \mu_{n+1}\right)}\right)^{\theta}\left(\sum_{n=0}^{\infty} 2^{-n-1}\|R(\cdot,(x, q), b)\|_{L_{p}\left(K, \mu_{n+1}\right)}\right)^{1-\theta} \\
& \leq c \cdot C_{1} \cdot \lambda\left(\sum_{n=0}^{\infty} 2^{-n-1}\|R(\cdot,(x, q), b)\|_{L_{1}\left(K, \mu_{n+1}\right)}\right)^{\theta}\left(2 \sum_{n=0}^{\infty} 2^{-n-1}\|R(\cdot,(x, q), b)\|_{L_{p}\left(K, \mu_{n}\right)}\right)^{1-\theta},
\end{aligned}
$$

where in $\left(^{*}\right)$ we used Holder's Inequality. Hence

$$
\begin{aligned}
\sum_{n=0}^{\infty} 2^{-n-1}\|R(\cdot,(x, q), b)\|_{L_{p}\left(K, \mu_{n}\right)} & \leq\left(c \cdot C_{1} \cdot \lambda\right)^{1 / \theta} 2^{\frac{1-\theta}{\theta}}\left(\sum_{n=0}^{\infty} 2^{-n-1}\|R(\cdot,(x, q), b)\|_{L_{1}\left(K, \mu_{n+1}\right)}\right) \\
& \leq\left(c \cdot C_{1} \cdot \lambda\right)^{1 / \theta} 2^{\frac{1-\theta}{\theta}} 2\left(\sum_{n=0}^{\infty} 2^{-n-1}\|R(\cdot,(x, q), b)\|_{L_{1}\left(K, \mu_{n}\right)}\right) .
\end{aligned}
$$

Note that

$$
\begin{aligned}
\|R(\cdot,(x, q), b)\|_{L_{1}(K, \bar{\mu})} & =\int_{K} R(\varphi,(x, q), b) d\left[\sum_{n=0}^{\infty} 2^{-n-1} \mu_{n}\right](\varphi) \\
& =\sum_{n=0}^{\infty} 2^{-n-1} \int_{K} R(\varphi,(x, q), b) d \mu_{n}(\varphi) \\
& =\sum_{n=0}^{\infty} 2^{-n-1}\|R(\cdot,(x, q), b)\|_{L_{1}\left(K, \mu_{n}\right)}
\end{aligned}
$$


So,

In particular,

$$
\sum_{n=0}^{\infty} 2^{-n-1}\|R(\cdot,(x, q), b)\|_{L_{p}\left(K, \mu_{n}\right)} \leq\left(2 c \cdot C_{1} \cdot \lambda\right)^{1 / \theta}\|R(\cdot,(x, q), b)\|_{L_{1}(K, \bar{\mu})} .
$$

$$
2^{-1}\|R(\cdot,(x, q), b)\|_{L_{p}\left(K, \mu_{0}\right)} \leq\left(2 c \cdot C_{1} \cdot \lambda\right)^{1 / \theta}\|R(\cdot,(x, q), b)\|_{L_{1}(K, \bar{\mu})} .
$$

Thus $C=2\left(2 c \cdot C_{1} \cdot \lambda\right)^{1 / \theta}$ is the desired constant.

\section{Recovering the Previous Extrapolation Theorems}

4.1. The Extrapolation Theorem for absolutely $p$-summing linear operators. Note that a continuous linear operator $T: X \rightarrow Y$ is absolutely $p$-summing if and only if it is $R S$-abstract $p$-summing with

$$
E=X \times X \text { and } G=\mathbb{R}
$$

and $K=B_{X^{*}}$, with the weak star topology, $\mathcal{H}(X ; Y)=\mathcal{L}(X ; Y)$ and $R$ and $S$ are defined by:

$$
\begin{gathered}
R: B_{X^{*}} \times(X \times X) \times \mathbb{R} \longrightarrow[0, \infty), R\left(\varphi,\left(x_{1}, x_{2}\right), \lambda\right)=\left|\varphi\left(x_{1}\right)\right| \\
S: \mathcal{L}(X ; Y) \times(X \times X) \times \mathbb{R} \longrightarrow[0, \infty), S\left(T,\left(x_{1}, x_{2}\right), \lambda\right)=\left\|T\left(x_{1}\right)\right\| .
\end{gathered}
$$

Since the hypotheses of Theorem 3.1 are straightforwardly satisfied, we recover the classical Extrapolation Theorem (Theorem 1.1).

4.2. The Extrapolation Theorem for Lipschitz $p$-summing maps. If $X=\left(X, d_{X}\right)$ and $Y=\left(Y, d_{Y}\right)$ are metric spaces, according to Farmer and Johnson [10, a map $T: X \longrightarrow Y$ is Lipschitz $p$-summing (notation $\left.T \in \Pi_{p}^{L}(X ; Y)\right)$ if there is a constant $C \geq 0$ such that, for all natural $n$ and $x_{1}, \ldots, x_{n}, y_{1}, \ldots, y_{n} \in$ $X$,

$$
\sum_{i=1}^{n} d_{Y}\left(T\left(x_{i}\right), T\left(y_{i}\right)\right)^{p} \leq C^{p} \sup _{f \in B_{X \#}} \sum_{i=1}^{n}\left|f\left(x_{i}\right)-f\left(y_{i}\right)\right|^{p},
$$

where $B_{X \#}$ is the unit ball of the Lipschitz dual $X^{\#}$ of $X$. The infimum of all such $C$ is denoted by $\pi_{p}^{L}$. Note that $T$ is Lipschitz $p$-summing if and only if it is $R S$-abstract $p$-summing with

$$
E=X \times X \text { and } G=\mathbb{R}
$$

and $K=B_{X^{\#}}$, which is a compact Hausdorff space in the topology of pointwise convergence on $Y, \mathcal{H}(X ; Y)$ is the set of all maps from $X$ to $Y$ and $R$ and $S$ are defined by:

$$
\begin{gathered}
R: B_{X^{\#}} \times(X \times X) \times \mathbb{R} \longrightarrow[0, \infty), R(f,(x, y), \lambda)=|f(x)-f(y)| \\
S: \mathcal{H}(X ; Y) \times(X \times X) \times \mathbb{R} \longrightarrow[0, \infty), S(T,(x, y), \lambda)=d_{Y}(T(x), T(y)) .
\end{gathered}
$$

As a consequence of Theorem 1.3 we have:

Theorem 4.1 (Farmer-Johnson). The following are equivalent for a mapping $T: X \longrightarrow Y$ between metric spaces:

(i) $T$ is Lipschitz p-summing.

(ii) There is a probability $\mu$ on $B_{X \#}$ and a constant $C \geq 0$ such that

$$
d_{Y}(T x, T y) \leq C\left(\int_{B_{X \#}}|f(x)-f(y)|^{p} d \mu(f)\right)^{1 / p} .
$$

It is well-known that if $Y$ is Banach space then $\left(\Pi_{p}^{L}(X ; Y), \pi_{p}^{L}\right)$ is also a Banach space and $\Pi_{p}^{L}$ has the ideal property. Moreover

$$
\begin{aligned}
S\left(j_{\mu},(x, y), \lambda\right) & =\left\|j_{\mu}(x)-j_{\mu}(y)\right\|_{L_{p}\left(B_{X}, \mu\right)}=\left(\int_{B_{X \#}}\left|j_{\mu}(x) f-j_{\mu}(x) f\right|^{p} d \mu(f)\right)^{1 / p} \\
& =\left(\int_{B_{X} \#}|f(x)-f(y)|^{p} d \mu(f)\right)^{1 / p}=\|R(\cdot,(x, y), \lambda)\|_{L_{p}\left(B_{X}, \mu\right)} .
\end{aligned}
$$

So, from Theorem 3.1 we recover the Extrapolation Theorem due to D. Chen and B. Zheng [7]: 
Theorem 4.2 (Extrapolation Theorem for Lipschitz $p$-summing operators). Let $1<r<p<\infty$ and let $X$ be a metric space. If

then, for any Banach space $Y$,

$$
\Pi_{p}^{L}\left(X ; \ell_{p}\right)=\Pi_{r}^{L}\left(X ; \ell_{p}\right)
$$

$$
\Pi_{p}^{L}(X ; Y)=\Pi_{1}^{L}(X ; Y) .
$$

A similar argument also shows that Theorem 1.2 holds, since conditions (1)-(5) are easily satisfied.

\section{REFERENCES}

[1] D. Achour, Multilinear extensions of absolutely $(p ; q ; r)$-summing operators, Rend. Circ. Mat. Palermo (in press). DOI: 10.1007/s12215-011-0054-2.

[2] G. Botelho, D. Pellegrino and P. Rueda, Pietsch's factorization theorem for dominated polynomials. J. Funct. Anal. 243 (2007), 257-269

[3] G. Botelho, D. Pellegrino and P. Rueda, A unified Pietsch Domination Theorem, J. Math. Anal. Appl. 365 (2010), 269-276.

[4] G. Botelho, D. Pellegrino and P. Rueda, Cotype and absolutely summing linear operators, Math. Z. 267 (2011), 1-7.

[5] J.A. Chávez-Domínguez, Duality for Lipschitz p-summing operators, J. Funct. Anal. 261 (2011), 387-407.

[6] J.A. Chávez-Domínguez, Lipschitz $(q ; p)$-mixing operators, Proc. Amer. Math. Soc. (in press).

[7] D. Chen and B. Zheng, Remarks on Lipschitz p-summing operators, Proc. Amer. Math. Soc. 139 (2011), $2891-2898$.

[8] J. Diestel, H. Jarchow and A. Tonge, Absolutely summing operators, Cambridge University Press, 1995.

[9] J. Diestel, A.M. Peralta and D. Puglisi, Sequential w-right continuity and summing operators, Math. Nachr. 284 (2011), 664-680.

[10] J. Farmer and W. B. Johnson, Lipschitz p-summing operators, Proc. Amer. Math. Soc. 137 (2009), $2989-2995$.

[11] D. J. H. Garling, Inequalities: A Journey into Linear Analysis, Cambridge University Press, New York, 2007.

[12] M.C. Matos, Nonlinear absolutely summing multilinear mappings between Banach spaces, Math. Nachr. 258 (2003), 71-89.

[13] B. Maurey, Théoremès de factorisation pour les opérateurs à valeurs dans les espaces $L_{p}$, Soc. Math. France, Asterisque 11, Paris, 1974.

[14] B. Mitiagin and A. Pełczyński, Nuclear operators and approximative dimensions, Proceedings International Congress of Mathematics, Moscow 1966.

[15] D. Pellegrino and J. Santos, On summability of nonlinear maps: a new approach, Math Z. (in press).

[16] D. Pellegrino and J. Santos, A general Pietsch Domination Theorem, J. Math. Anal. Appl. 375 (2011), 371-374.

[17] D. Pellegrino, J. Santos and J.B. Seoane-Sepúlveda, Some techniques on nonlinear analysis and applications. Adv. Math. 229 (2012), 1235-1265.

[18] A. Pietsch, Absolut p-summierende Abbildungen in normieten Raumen, Studia Math. 27 (1967), 333-353.

Departamento de Matemática,

Universidade Federal da Paraíba,

58.051-900 - JoÃo PessoA, Brazil.

E-mail address: pellegrino@pq.cnpq.br and dmpellegrino@gmail.com

Departamento de Matemática,

Universidade Federal de Sergipe,

49.500-000 - Itabaiana, Brazil.

E-mail address: joedsonsr@yahoo.com.br

Departamento de Análisis Matemático,

Facultad de Ciencias Matemáticas,

Plaza de Ciencias 3 ,

Universidad Complutense de Madrid,

MADRID, 28040, SPAIN.

E-mail address: jseoane@mat.ucm.es 